\title{
Uma análise dos sites de governos eletrônicos no Brasil sob a ótica dos usuários dos serviços e sua satisfação
}

\author{
leda Pelógia Martins Damian \\ Universidade de São Paulo \\ Edgard Monforte Merlo \\ Universidade de São Paulo
}

\begin{abstract}
Os sites de governo eletrônico (e-gov) têm sido muito analisados do ponto de vista dos aspectos técnicos, existindo poucos estudos que os avaliem do ponto de vista dos usuários dos serviços oferecidos por estes sites. Este estudo propôs um modelo de análise dos serviços de sites de egov avaliados com a técnica de análise de conteúdo e identificados os níveis de atendimento dos atributos do modelo. As principais contribuições do estudo são: uma crítica das deficiências verificadas nos principais sites analisados e uma proposição de um modelo abrangente que facilite a orientação para o atendimento das principais dimensões a serem analisadas, quais sejam: eficiência, realização, disponibilidade do sistema, privacidade, site e sinergia. Do ponto de vista gerencial, o estudo contribuiu com um modelo de fácil aplicação que pode auxiliar a identificar os aspectos mais importantes dos serviços de e-gov.
\end{abstract}

Palavras-chave: governo eletrônico; serviços; satisfação; modelo; site.

Un análisis de los sitios de gobierno electrónico en Brasil desde la perspectiva de los usuarios del servicio y su satisfacción

Los sitios web de gobierno electrónico (e-gob) han sido muy analizada desde el punto de vista de los aspectos técnicos, hay pocos estudios que evalúan la misma desde el punto de vista de los usuarios de los servicios ofrecidos por estos sitios. Este estudio propone un modelo de análisis de los servicios de los sitios de e-gob evaluaron la técnica de análisis de contenido y los niveles de servicio de los atributos del modelo. Las principales aportaciones del estudio son: una crítica de las debilidades de los sitios clave analizados y una propuesta de un modelo integral que facilita la orientación a satisfacer las principales dimensiones a tener en cuenta, a saber: la eficiencia, la realización, la disponibilidad del sistema, la privacidad, sitio y la sinergia. Desde el punto de vista gerencial, el estudio aportó un modelo de fácil aplicación que puede ayudar a identificar los aspectos más importantes de los servicios de e-gob.

Palabras Clave: gobierno electrónico; servicios; satisfacción; modelo; sitio.

Artigo recebido em 25 abr. 2012 e aceito em 16 abr. 2013.

Rev. Adm. Pública - Rio de Janeiro 47(4):877-99, jul./ago. 2013 


\begin{abstract}
An analysis of electronic government sites in Brazil from the perspective of service users and their satisfaction

The e-government websites (e-gov) have been much analyzed from the point of view of the technical aspects; there are few studies that evaluate the same from the point of view of the users of the services offered by these sites. This study proposed a model of analysis services sites e-gov evaluated the technique of content analysis of these identified sites and service levels of the attributes of the model. The main contributions of the study are: a critique of the weaknesses in key sites analyzed and a proposal of a comprehensive model that facilitates the orientation to meet the main dimensions to be considered, namely: efficiency, fulfillment, system availability, privacy, site and synergy. From the managerial point of view, the study contributed a model of easy application that may help identify the most important aspects of e-gov services.
\end{abstract}

KEYWORDs: electronic government; services; satisfaction; model; site.

\title{
1. Introdução
}

Uma das mais significativas inovações na tecnologia da informação foi a criação e o desenvolvimento contínuo da internet. A internet aumenta a flexibilidade da comunicação, reduz o custo, permite a troca de grandes quantidades de informação instantaneamente, independentemente da distância geográfica. Devido a essas vantagens, os governos estão cada vez mais utilizando a internet para se comunicar e realizar transações com cidadãos, o que é comumente referido como governo eletrônico (Mcneal et al. 2003).

Mais do que acrescentar uma página na internet para a prestação de serviço, o governo eletrônico deve envolver: (a) uma reengenharia no modo de trabalho dos governos; (b) acesso direto com os cidadãos, fornecedores e diversos clientes internos dentro do governo; (c) realização de um trabalho mais eficiente, que cruza as fronteiras dos departamentos, o que permite uma organização virtual estruturada focada nos clientes e em suas necessidades; e (d) reexame do papel do governo e a implementação de suas políticas, para que novos modelos de entrega de serviços e parcerias com o setor privado sejam possíveis (Almeida, 2002).

Em relação aos benefícios do e-gov, Diniz (2000) afirmou que os serviços públicos podem ser democratizados e melhorados por meio do uso da internet pelo setor público, facilitando o relacionamento e as transações entre o cidadão e o governo: os serviços públicos oferecidos pela internet podem ser obtidos de forma mais rápida, o que aumentaria a eficácia governamental no atendimento aos cidadãos.

Os efeitos positivos do governo eletrônico que, de acordo com Hoeschl (2003), podem ser indicados como objetivos estratégicos são: melhoria da qualidade, segurança e rapidez dos serviços para o cidadão; simplificação dos procedimentos e diminuição da burocracia; avanço da cidadania; democracia da informação; transparência e otimização 
das ações do governo; educação para a sociedade da informação; facilidade de acessar o governo; integração das informações para o cidadão; geração de empregos na iniciativa privada; otimização no uso e aplicação dos recursos disponíveis; integração entre os órgãos do governo; aproximação com o cidadão; desenvolvimento do profissional do serviço público; aperfeiçoamento dos modelos de gestão pública; universalização do acesso à informação.

Diante deste contexto, esta pesquisa foi norteada pela seguinte questão: como poderia ser um modelo para análise dos serviços de sites de governos eletrônicos, do ponto de vista dos cidadãos?

Conforme Costa (2008), no Estado brasileiro ocorreram três grandes reformas, a de 1937, que se deu logo após o Estado Novo e que estruturou o moderno estado capitalista com suas atividades estruturantes; a de 1967, que aconteceu após a revolução de 1964 (DecretoLei no 200), que possibilitou a reorganização do Estado e a construção de mecanismos de planejamento e financiamento dos gastos estatais. Finalmente, tem-se a de 1995, conhecida por incorporar as preocupações com a desburocratização e desestatização, na qual destacouse, também, a preocupação com a racionalidade gerencial dentro do setor estatal. Esta última reforma utilizou cinco diretrizes principais, quais sejam: a institucionalização, a racionalização, a flexibilização, a publicização e a desestatização, que incorporava as reflexões críticas da New Public Management (Osborne e Gaebler, 1997). Neste contexto de reforma ocorreu o desenvolvimento da implantação do e-gov no Estado brasileiro em seus diversos níveis de governo (municipal, estadual e federal).

O intuito de avaliar os serviços dos sites de e-gov dos estados de São Paulo, Paraná, Minas Gerais, Rio Grande do Sul e Santa Catarina deve-se ao fato de que estes representam 50\% dos sites nacionais de e-gov disponíveis, do ponto de vista de bytes transmitidos, conforme demonstrado no gráfico seguinte.

Além da representatividade dos sites escolhidos, conforme estudos de Diniz (2000), Fernandes e Afonso (2001) e Arakaki (2008), os referidos estados possuíam as iniciativas de e-gov mais avançadas, relativamente aos demais estados brasileiros.

Foram analisados os serviços dos e-gov estaduais, devido ao interesse do pesquisador por este aspecto. Deve-se observar que, no Brasil, no relacionamento dos cidadãos com o poder público, o Estado desempenha um papel de grande importância, ao organizar os serviços públicos que chegam diretamente aos usuários, muitas vezes (por questão de escala) integrando e articulando os serviços municipais e estaduais.

Ao analisar a literatura existente sobre o assunto, foi possível verificar que a maioria dos estudos estava voltada para análise de aspectos e critérios que poderiam auxiliar na avaliação de sites de e-gov do ponto de vista de sistemas ou do ponto de vista de banco de dados (Hoeschl, 2003; Villela, 2003). 
Participação das regiões na composição da web governamental por UF

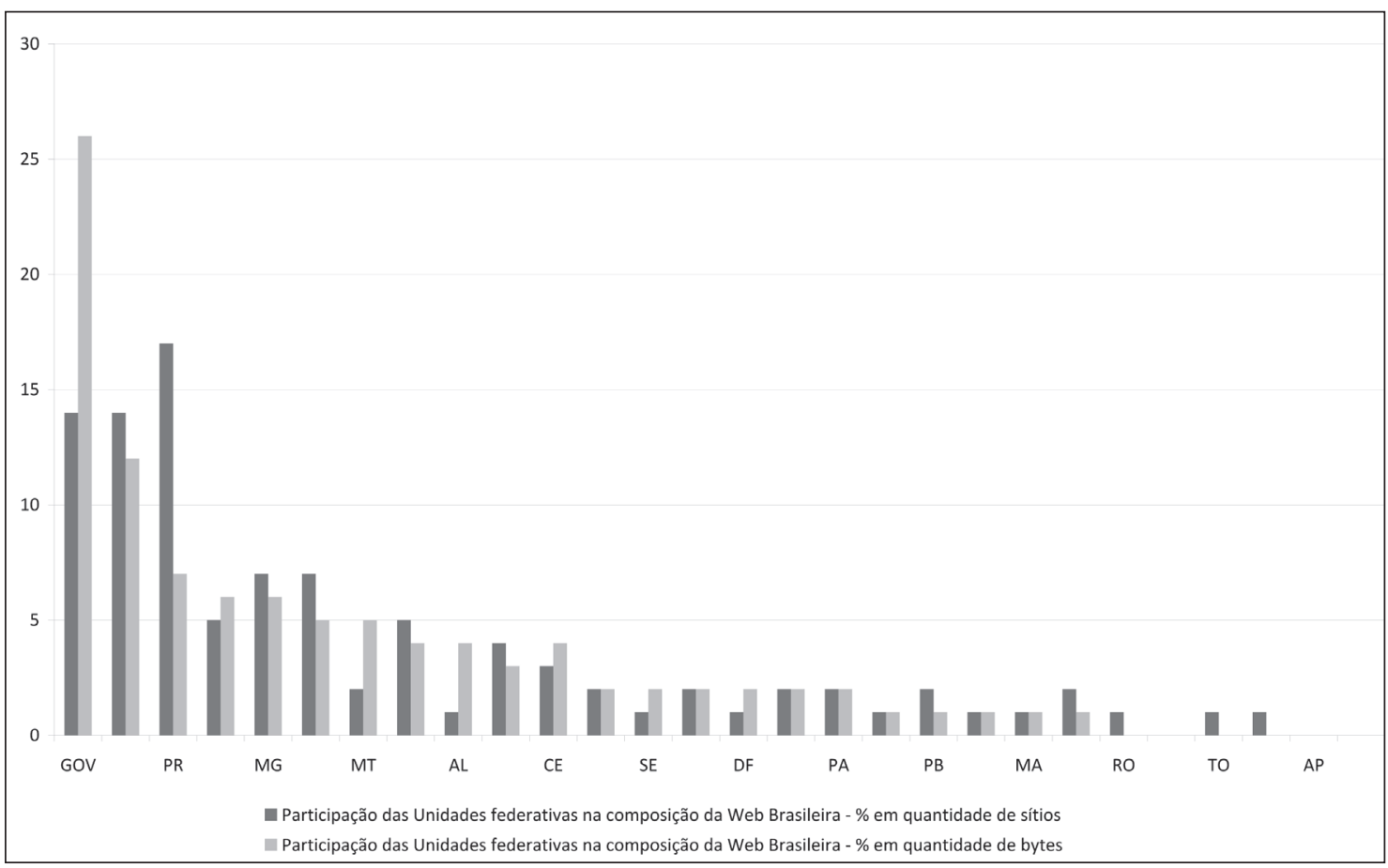

Fonte: CGI.br (2011).

\section{Referencial teórico}

\section{A relação entre os negócios eletrônicos e o governo eletrônico}

Os negócios eletrônicos podem ser definidos, segundo Lin (2008), como uma aplicação baseada na internet utilizada por uma empresa para integrar suas atividades de negócios internos, processos, sistemas de informação (SI) e a realização de transações comerciais com parceiros comerciais.

Complementando as definições acima, para Gonçales e Ferreira (2010) o e-business representa uma série de modalidades específicas de negócios realizados pela internet entre entidades que podem ser tanto pessoas físicas como jurídicas, dentre as quais podem ser citados o e-service, serviços diversos cuja contratação é oferecida por empresas via internet, como entregas, compras; o e-learning, conhecido como educação a distância (EAD); o e-banking, serviços bancários, pagamentos de contas, depósitos, transferências; o e-commerce, o comércio eletrônico, a mais conhecida e utilizada de todas as modalidades de negócios eletrônicos; 
e o e-governo, serviços governamentais oferecidos eletronicamente à população, que é a modalidade de interesse deste estudo e, portanto, será descrita em detalhes adiante.

\section{Governo eletrônico}

A expressão governo eletrônico é, de acordo com Sanchez e Araújo (2003), utilizada para designar o conjunto de atividades que os órgãos governamentais realizam por meio da tecnologia de informação e comunicação (TIC) e não se restringe a distribuir e instalar computadores e sistemas de informação no estado, para uso interno; mas trabalhar para efetivar, por meio da tecnologia, uma relação mais direta, transparente e participativa entre as instituições governamentais e o cidadão.

Para West (2004), o e-gov refere-se à entrega de informações do governo e serviços online por meio da internet ou outros meios digitais, disponível 24 horas por dia, sete dias por semana, e libera os cidadãos a procurar informação de acordo com sua própria conveniência e não apenas quando um escritório do governo está aberto.

Para Diniz e colaboradores (2009), o e-gov pode ser considerado uma evolução do uso estratégico das TICs como elemento viabilizador de um novo modelo de gestão pública, constituindo-se em uma das principais formas de modernização do Estado, mudando a maneira pela qual o governo interage com o cidadão, empresas e outros governos.

Um programa de e-gov deveria, de acordo com Arakaki (2008), contemplar ações mais abrangentes do que se restringir a projetos de portais de governos para a internet: ele deveria englobar a ideia de desenvolver um governo que realizasse uma aproximação entre o cidadão e o governo por meio do uso das TICs com o objetivo de melhorar os serviços e a informação oferecidos a todos os cidadãos, aumentar a eficiência e a eficácia da gestão pública e incrementar a transparência do setor público e a participação cidadã.

Apesar de ser um objeto com aspecto tecnológico, o e-gov, ainda na visão da autora citada, poderia ser analisado sob outra ótica, a da comunicação, uma vez que o portal é um meio de comunicação. O e-gov pode ainda ser visto como uma iniciativa para modernizar a administração pública, tornando-se o resultado do processo de evolução do modelo de gestão burocrático para o gerencial, que passa a priorizar os fins, que, neste caso específico, são os cidadãos.

Essa visão de e-gov proposta por Arakaki (2008), em que as TICs são um meio e não o fim, ou seja, utilizar tecnologia da informação para modernizar o governo de modo que este possa oferecer serviços eletrônicos que realmente atendam as necessidades dos cidadãos e não apenas fazer uso da internet para informatizar processos burocráticos, será a definição norteadora de e-gov deste estudo, uma vez que o foco da análise é o ponto de vista do cidadão e os aspectos funcionais (explicados adiante) envolvidos nos projetos de e-gov e não seus aspectos tecnológicos.

Uma das maiores vantagens do e-gov ressaltada por Endler (2000) é a diminuição do volume de pessoas que procuram e congestionam os órgãos públicos. Para o autor, os ser- 
viços públicos na internet devem proporcionar: (1) redução de gastos com infraestrutura e funcionalismo; (2) maior sincronia no processo alcançada pelo uso intensivo da tecnologia da informação; (3) redução de perdas por transporte já que estas podem ser quase totalmente eliminadas com o uso de infraestruturas de telecomunicações seguras e estáveis; (4) redução de perdas por espera já que o serviço estará ao alcance da mão, disponível sempre que for solicitado.

Apesar dos inúmeros benefícios oferecidos pelo e-gov, muitas barreiras ainda precisam ser vencidas. Em sua pesquisa, West (2004) identificou uma série de dificuldades a serem superadas, como a preocupação dos cidadãos com relação à privacidade e à segurança dos sites do governo; a falta de recursos para atender as necessidades de populações especiais, como os deficientes e pessoas que não falam inglês; a indisponibilidade de computadores e da internet; a necessidade de educar o cidadão quanto à existência de serviços e informações on-line; e o custo da infraestrutura do governo eletrônico.

Outro desafio, de acordo com Villela (2003), é a necessidade de atuação dos governos em um novo cenário que é marcado por novas exigências impostas pelos cidadãos e pela própria multiplicidade e velocidade de desenvolvimento das soluções tecnológicas, que acabam impondo aos gestores públicos a realização de uma análise ainda mais criteriosa dos objetivos, estratégias e metas dos governos para o uso das inúmeras possíveis soluções tecnológicas.

Como um dos objetivos desta pesquisa foi realizar uma análise dos cinco principais sites de e-gov brasileiros e sua relação com os cidadãos, a seguir são discutidos a implantação e o desenvolvimento do governo eletrônico nos estados pesquisados.

\section{Governo eletrônico nos estados analisados}

Muitos dos serviços eletrônicos paulistas estão sob a coordenação da Companhia de Processamento de Dados do Estado de São Paulo (Prodesp) e algumas iniciativas merecem ser destacadas, como é o caso do Poupatempo, AcessaSP, e-poupatempo.

O site escolhido para as análises referentes aos serviços de e-gov paulista é o PoupaTempo ${ }^{1}$ porque representa um programa muito conhecido e utilizado no estado de São Paulo. Além disso, é um programa com uma característica fundamental: existe tanto em meio físico quanto no virtual.

O Paraná, ${ }^{2}$ segundo Fernandes e Afonso (2001), desenvolveu um programa específico para a implantação do e-gov no estado chamado Rede Cidadão, com o objetivo de disponibilizar, por meio da internet, os serviços prestados pelos órgãos da administração pública estadual, com o intuito de reduzir o deslocamento das pessoas e as filas nos balcões de aten-

\footnotetext{
${ }^{1}$ Disponível em: <www.poupatempo.sp.gov.br>. Acesso em: 19 maio 2011.

${ }^{2}$ Disponível em: <www.pr.gov.br>. Acesso em: 19 maio 2011.
} 
dimento. Esse programa evoluiu e deu origem ao e-Paraná que confere mais ênfase à questão da universalização do acesso.

Seguindo os mesmos critérios detalhados nos tópicos anteriores, o programa de serviços de e-gov paranaense a ser analisado será o e-Paraná.

O estado gaúcho, segundo a fonte Professor Digital (2011), foi pioneiro em ações de e-gov no Brasil: as atividades foram iniciadas há mais de 30 anos por meio da Companhia de Processamento de Dados do Estado do Rio Grande do Sul (Procergs).

O estado oferece por meio do TudoFácil Eletrônico ${ }^{3}$ um catálogo com mais de 700 informações e serviços. Por meio dessas ações, os principais objetivos que se pretende alcançar são: transparência, inovação, controle social e integração estado e cidadão (Professor Digital, 2011).

Baseando-se nos critérios de escolha adotados nos tópicos anteriores e na exposição feita acima, o programa gaúcho de serviços de e-gov a ser analisado neste estudo é o TudoFácil.

O Estado de Minas Gerais ${ }^{4}$ desenvolveu, de acordo com Fernandes e Afonso (2001), um programa chamado Minas Interativa cuja finalidade foi colocar todos os serviços prestados pelo governo mineiro em um único endereço e determinar a divulgação, via internet, de dados da execução orçamentária. Os objetivos gerais do programa são: atendimento 24 horas, todos os dias, ao cidadão; prestação de serviços em todo o estado; redução do tempo de atendimento; controle dos serviços prestados; eliminação de intermediários; transparência nos serviços e consequente redução de custos para o governo.

O Governo de Minas Gerais oferece à sua população a Unidade de Atendimento Integrado (UAI) disponibilizando serviços públicos com foco na desburocratização e no atendimento de seus usuários. As UAIs reúnem, em um mesmo espaço físico, vários órgãos e entidades integradas (UAI, 2011).

Com o intuito de fazer com que as análises realizadas neste estudo trouxessem importantes contribuições para a proposição do modelo de análise dos serviços de sites de e-gov, buscou-se analisar, em cada estado envolvido na pesquisa, um site de e-gov que oferecesse serviços aos cidadãos que, no caso do Estado de Minas Gerais, foi o UAI. ${ }^{5}$

O portal de Santa Catarina, ${ }^{6}$ de acordo com Fernandes e Afonso (2001), tem se destacado no contexto de e-gov nacional devido a seus esforços de adaptar as novas tecnologias às práticas governamentais, como no caso da criação e desenvolvimento da Net Escola, um conjunto de inovações tecnológicas com o objetivo de democratizar as informações educacionais da rede estadual de ensino.

\footnotetext{
${ }^{3}$ Disponível em: <www.tudofacil.rs.gov.br>. Acesso em: 19 maio 2011.

${ }^{4}$ Disponível em: <www.mg.gov.br>. Acesso em: 19 maio 2011.

${ }^{5}$ Disponível em: <www.mg.gov.br/governomg/portal/m/governomg/acesso-rapido/10652-uai/10652/5309>, também disponível em: <www.uai.mg.gov.br>. Acesso em: 19 maio 2011.

${ }^{6}$ Disponível em: <www.sc.gov.br>. Acesso em: 19 maio 2011.
} 


\section{Avaliação de governo eletrônico: aspectos de qualidade e de marketing}

Este estudo abordou a questão da qualidade e do marketing (no sentido de relacionamento com os usuários dos serviços oferecidos). Apesar de os autores considerarem que os interesses políticos são relevantes, sua incorporação no processo de avaliação poderia comprometer a objetividade no tratamento dos aspectos dos serviços oferecidos pelos governos.

Mais especificamente em relação aos serviços públicos na internet, Endler (2000) indicou que estes basicamente processam informações que estarão ao alcance da mão proporcionando investimentos em serviços do tipo self-service. Neste caso, continua o autor, deve-se ter uma forte atenção para o layout da interface com o usuário, pois é nesse ambiente virtual que devem ocorrer o relacionamento entre o cidadão e as instituições públicas.

Parasuraman, Zeithaml e Malhotra (2005) se propuseram a conceituar, construir, refinar e testar uma escala de vários itens para medir a qualidade do serviço oferecido por sites. Os autores desenvolveram a escala denominada E-S-QUAL, composta por quatro dimensões e 22 itens.

As quatro dimensões da escala E-S-QUAL são as seguintes:

1. Eficiência: A velocidade de acessar e a facilidade de usar o site;

2. Realização: Em que medida o site promete e cumpre a entrega dos pedidos e a disponibilidade de itens;

3. Disponibilidade do sistema: O funcionamento técnico correto do site;

4. Privacidade: Em que grau o site é seguro e protege as informações do cliente.

Apesar de existirem importantes trabalhos focados na qualidade dos serviços eletrônicos, apenas a ferramenta E-S-QUAL de Parasuraman, Zeithaml e Malhotra (2005) foi desenvolvida especificamente para o contexto eletrônico, tendo como foco a qualidade dos serviços eletrônicos, considerando apenas os encontros rotineiros, ou seja, apenas aqueles encontros que realmente deveriam acontecer. Contudo, dado que a ferramenta não avalia elementos importantes como sinergia e aspectos motivacionais, foram utilizados neste estudo, de modo complementar, outros métodos de avaliação (Modelo dos 4S e os fatores influenciadores da experiência na Web - Fiew). ${ }^{7}$ Estes dois modelos, com uma maior ênfase nos elementos de marketing, que também compõem o aqui denominado aspecto funcional, serão discutidos no próximo tópico.

Para que as estratégias sejam apropriadas ao mercado virtual, faz-se necessário compreender o que envolve uma experiência na web. Essa experiência pode ser definida como a impressão que o consumidor tem da empresa on-line e esta impressão é resultado de sua exposição a uma combinação de ferramentas virtuais de marketing sob o controle direto do

\footnotetext{
${ }^{7} \mathrm{O}$ acrônimo Fiew será utilizado como forma de síntese para se referir aos fatores influenciadores da experiência na Web de Constantinides (2004).
} 
gestor, susceptíveis de influenciar o comportamento do consumidor on-line. Essa experiência compreende elementos como pesquisar, navegar, encontrar, selecionar, comparar e avaliar informações bem como interagir e transacionar com a organização on-line. A impressão total do cliente virtual e suas ações são influenciadas pelo design, eventos, emoções, atmosfera e outros elementos experimentados durante a interação com um site, elementos destinados a induzir a boa vontade do cliente e a afetar o resultado final desta interação on-line. É importante ressaltar que a experiência na web é essencial tanto para sites de produtos ou serviços, quanto para sites com foco nos clientes interessados em conteúdo informativo, sites agindo como intermediários on-line e em geral a todos os tipos de empreendimentos que concorrem com a internet para obter a atenção do público on-line (Constantinides, 2002b, 2004). Assim, a experiência na web é, também, fundamental para os sites que fornecem serviços de governo eletrônico, objeto de estudo desta pesquisa, e será, portanto, um aspecto a ser explorado com mais detalhes e considerado no desenvolvimento do modelo proposto neste estudo.

O principal meio de entrega da experiência Web é, de acordo com Constantinides (2004), o site corporativo, e os sites que entregam excelentes experiências na web não são projetados apenas para tratar das necessidades e expectativas do cliente: eles devem ser vistos como instrumentos vitais de atendimento ao cliente, em vez de simplesmente brochuras online ou catálogos de produtos da empresa.

Existem três grandes fatores que, segundo Constantinides (2004), influenciam a experiência na web (Fiew) e que são diretamente associados ao sucesso ou ao fracasso dos sites:

1. Fatores de funcionalidade: seus componentes são usabilidade e interatividade e influenciam diretamente a experiência do consumidor on-line.

2. Fatores psicológicos: diz respeito à confiança on-line, pois a distância física, a falta de contato pessoal e o anonimato da internet podem aumentar a ansiedade dos consumidores.

3. Fatores de conteúdo: são divididos em duas categorias - estética e mix de marketing. Os elementos que compõem a estética comunicam a atmosfera do site, que é importante para atrair os clientes on-line para pararem, analisarem e, possivelmente, interagirem com o site.

Além da importância dos fatores de funcionalidade, psicológicos e de conteúdo na experiência na Web, seus componentes se assemelham, em muitos casos, com as dimensões da escala E-S-QUAL discutida anteriormente e, portanto, também serão considerados na proposição do modelo a ser desenvolvido nesta pesquisa.

As diferenças essenciais entre os comércios físicos e virtuais fazem, segundo Constantinides (2002b), com que os princípios de marketing precisem ser revistos para melhor se adequarem aos ambientes virtuais. O autor afirmou que o tradicional mix de marketing composto pelos 4 Ps (product, price, promotion e place) é incompatível com o contexto eletrônico e propôs o mix de marketing para web que identifica os elementos críticos de marketing para tal ambiente, composto pelos 4 Ss:

(1) Escopo (Scope): relacionado à estratégia e aos objetivos, com o intuito de pesquisar as diretrizes das decisões tomadas em quatro áreas: os objetivos estratégicos e operacionais 
do empreendimento on-line; a definição de mercado, que inclui a medição do mercado potencial e a identificação/classificação dos competidores potenciais, visitantes e clientes do site; o grau de preparo da organização para o comércio eletrônico e o papel estratégico do comércio eletrônico para a organização.

(2) Site: com o objetivo de compreender a experiência com a web, questiona, principalmente, o que o consumidor espera do site, por que esse consumidor fará uso do site e o que o motiva a voltar.

(3) Sinergia (Synergy): trata dos aspectos de integração classificados em três categorias: (a) integração de linha de frente, que se refere às estratégias de comunicação e distribuição da organização convencional; (b) integração de retaguarda, que envolve um conjunto de aspectos como a integração das atividades de suporte físico do comércio eletrônico dentro dos processos organizacionais existentes, a integração com sistemas mais antigos (legados) e integração da operação on-line dentro do sistema de valor da empresa; e (c) integração com terceiros, que significa criar uma rede de parceiros que prestarão assistência comercial, logística e outras atividades do site.

(4) Sistema (System): engloba tecnologia, requerimentos técnicos, serviços e administração do site.

Entre as várias alternativas de modelos de mix de marketing para os ambientes virtuais, o modelo dos 4 Ss (web-marketing model, WMM) é, segundo Wang, Wang e Yao (2005), utilizado com maior frequência, viável para as empresas que vendem serviços on-line e mais adequado que o modelo dos 4 Ps.

O quadro a seguir demonstra, de maneira resumida, os trabalhos apresentados neste tópico, bem como suas principais contribuições/críticas.

Quadro 1

\section{Principais contribuições e críticas dos modelos apresentados}

\begin{tabular}{|c|c|c|c|}
\hline Autor & Estudo & Principais contribuições & Críticas \\
\hline $\begin{array}{l}\text { Parasuraman, } \\
\text { Zeithaml e } \\
\text { Malhotra (2005) }\end{array}$ & $\begin{array}{l}\text { Desenvolver uma escala } \\
\text { para medir a qualidade } \\
\text { do serviço oferecido por } \\
\text { sites. }\end{array}$ & $\begin{array}{l}\text { Desenvolveram duas escalas: a E-S-QUAL } \\
\text { e a E-Rec-S-QUAL. }\end{array}$ & $\begin{array}{l}\text { Não mensurar aspectos } \\
\text { motivacionais e de sinergia } \\
\text { (comunicação, FAQ etc.), } \\
\text { importantes no contexto eletrônico. }\end{array}$ \\
\hline $\begin{array}{l}\text { Collier e } \\
\text { Bienstock } \\
(2006)\end{array}$ & $\begin{array}{l}\text { Avaliar a qualidade dos } \\
\text { serviços eletrônicos. }\end{array}$ & $\begin{array}{l}\text { A avaliação da qualidade dos senviços } \\
\text { eletrônicos é baseada no processo interativo } \\
\text { que ocorre on-line, no resultado de como o } \\
\text { produto ou serviço é entregue e na maneira } \\
\text { pela qual as falhas são tratadas. }\end{array}$ & $\begin{array}{l}\text { Utilizar o mesmo instrumento de } \\
\text { mensuração para avaliar serviços } \\
\text { rotineiros e os não rotineiros (de } \\
\text { recuperação). }\end{array}$ \\
\hline
\end{tabular}

Fonte: Elaborado pelos autores.

Devido ao papel de destaque desempenhado pelo mix de marketing e a sua adequação ao contexto virtual, os elementos do WMM também foram considerados, de modo comple- 
mentar, no modelo proposto para análise dos serviços de sites de e-gov desenvolvido neste estudo, conforme será detalhado adiante.

\section{Modelo para análise dos serviços de sites de governo eletrônico}

As dimensões e os aspectos contemplados pela escala E-S-QUAL desenvolvida por Parasuraman, Zeithaml e Malhotra (2005), pelo modelo dos 4 Ss de Constantinides (2002a) e pelos fatores influenciadores da experiência na Web (Fiew) identificados por Constantinides (2004) serão utilizados neste estudo de modo complementar, com o objetivo de analisar os serviços de sites de e-gov do ponto de vista do cidadão, usuário dos serviços avaliados.

Os aspectos que foram avaliados em cada dimensão, bem como os modelos de onde essas dimensões foram extraídas, podem ser visualizados no quadro 2.

$$
\text { Quadro } 2
$$

\section{Aspectos que foram avaliados}

\begin{tabular}{|c|c|c|}
\hline Dimensão & Origem da dimensão & Aspectos a serem analisados \\
\hline Eficiência & $\begin{array}{l}\text { E-S-QUAL } \\
\text { Fiew/Funcionalidade }\end{array}$ & $\begin{array}{l}\text { - Velocidade para acessar o site; } \\
\text { - Facilidade para utilizar o site; } \\
\text { - Facilidade de interação do site; } \\
\text { - Conveniências de usar o site; } \\
\text { - Serviços personalizados; } \\
\text { - Serviços de qualidade. }\end{array}$ \\
\hline Realização & $\begin{array}{l}\text { E-S-QUAL } \\
\text { Fiew/Conteúdo }\end{array}$ & $\begin{array}{l}\text { - O site apresenta claramente os serviços oferecidos; } \\
\text { - O site cumpre com os serviços oferecidos; } \\
\text { - O site permite acompanhar processos em andamento. }\end{array}$ \\
\hline $\begin{array}{l}\text { Disponibilidade } \\
\text { do sistema }\end{array}$ & E-S-QUAL & $\begin{array}{l}\text { - O site não apresenta problemas de indisponibilidade; } \\
\text { - Os links do site funcionam corretamente; } \\
\text { - O site não para de funcionar ("trava") durante o uso. }\end{array}$ \\
\hline Privacidade & $\begin{array}{l}\text { E-S-QUAL } \\
\text { Fiew/Psicológicos }\end{array}$ & $\begin{array}{l}\text { - As transações exigem senhas; } \\
\text { - O site disponibiliza política de segurança e privacidade; } \\
\text { - O site sinaliza a existência de mecanismos de segurança. }\end{array}$ \\
\hline Site & $\begin{array}{l}4 \text { Ss } \\
\text { Fiew/Conteúdo }\end{array}$ & $\begin{array}{l}\text { - O site oferece o que o cidadão esperava; } \\
\text { - O site motiva o cidadão a voltar; } \\
\text { - O site estimula o cidadão a utilizá-lo. }\end{array}$ \\
\hline Sinergia & $\begin{array}{l}4 \text { Ss } \\
\text { Fiew/Conteúdo }\end{array}$ & $\begin{array}{l}\text { - O site fornece informações de qualidade; } \\
\text { - O site oferece opções de comunicação (e-mail, telefone); } \\
\text { - As opções de comunicação funcionam; } \\
\text { - O site disponibiliza FAQ. }\end{array}$ \\
\hline
\end{tabular}

Fonte: Elaborado pelos autores. 
Conforme pode ser observado no quadro 2, o que se pretende analisar diz respeito aos aspectos funcionais da avaliação de governo eletrônico, envolvendo elementos da qualidade do serviço e do mix de marketing do ponto de vista do cidadão.

Partindo da premissa de avaliar os serviços de e-gov de acordo com seus aspectos funcionais e do ponto de vista do cidadão, dois fatores do modelo dos 4 Ss, escopo e sistema, não serão considerados. O escopo não será considerado porque diz respeito à estratégia e aos objetivos da empresa, ou seja, o foco deste fator é a organização e não o cidadão e, portanto, não faz parte da abrangência deste estudo. O fator sistema, por sua vez, também não foi utilizado por envolver aspectos diretamente relacionados a TI como requerimentos técnicos, serviços e administração do site. É prudente ressaltar que as dimensões escopo e sistema do modelo 4 Ss não serão utilizadas no desenvolvimento do modelo proposto por este estudo por questões de objetivos de pesquisa, o que não significa que tais dimensões não sejam importantes para outras situações.

Com um foco diferente dos modelos até então apresentados, Valdés e colaboradores (2011) desenvolveram um modelo com o intuito de avaliar o nível de maturidade do e-gov. Para tanto, os autores criaram cinco níveis de maturidade (inicial, em desenvolvimento, definido, realizado e otimizado) que deveriam ser avaliados de acordo com os domínios de alavancagens e suas respectivas áreas-chave de domínio, conforme representados no quadro 3.

Quadro 3

Maturidade organizacional em termos de capacidade de áreas prioritárias de domínio

\begin{tabular}{|c|c|c|c|c|c|c|}
\hline \multirow[b]{2}{*}{$\begin{array}{l}\text { Domínio de } \\
\text { Alavancagem }\end{array}$} & \multirow[b]{2}{*}{ Área-chave de domínio } & \multicolumn{5}{|c|}{ Nível de Maturidade Organizacional } \\
\hline & & 1. Inicial & $\begin{array}{c}\text { 2. Em } \\
\text { desenvolvi- } \\
\text { mento }\end{array}$ & 3. Definido & 4. Realizado & 5. Otimizado \\
\hline \multirow{4}{*}{$\begin{array}{l}\text { Estratégia de } \\
\text { E-Gov }\end{array}$} & Visão, estratégia e política & \multirow{2}{*}{\multicolumn{2}{|c|}{2}} & \multirow[t]{2}{*}{3} & 4 & 5 \\
\hline & Arquitetura da estratégia da em- & & & & 3 & 4 \\
\hline & presa & \multirow{2}{*}{\multicolumn{2}{|c|}{2}} & \multirow[t]{2}{*}{3} & 4 & 5 \\
\hline & Gerenciamento e organização de TI & & & & & \\
\hline \multirow{4}{*}{$\begin{array}{l}\text { Governança } \\
\text { de TI }\end{array}$} & Arquitetura de $\mathrm{TI}$ & \multirow{4}{*}{\multicolumn{2}{|c|}{2}} & 2 & 3 & 4 \\
\hline & Gestão de portfólio e risco & & & 3 & 4 & 5 \\
\hline & Prestação de serviços de TI & & & 2 & 3 & 4 \\
\hline & Utilização de ativos & & & 2 & 3 & 4 \\
\hline \multirow{6}{*}{$\begin{array}{l}\text { Gestão de } \\
\text { Processos }\end{array}$} & Gestão de processos de negócios & & & 2 & 3 & 4 \\
\hline & Gestão de desempenho & & & 2 & 3 & 4 \\
\hline & Serviços aos cidadãos e empresas & & 2 & 3 & 4 & 5 \\
\hline & Interoperabilidade & & 2 & 3 & 4 & 5 \\
\hline & Observância & & 2 & 3 & 4 & 5 \\
\hline & Qualidade e garantia de segurança & & & 3 & 3 & 4 \\
\hline \multirow{5}{*}{$\begin{array}{l}\text { Organização } \\
\text { e as Pessoas }\end{array}$} & Infraestrutura e ferramentas de & \multirow{2}{*}{\multicolumn{2}{|c|}{2}} & \multirow[t]{2}{*}{3} & 4 & 5 \\
\hline & e-Gov & & & & 3 & 4 \\
\hline & Gestão do conhecimento & \multirow{3}{*}{\multicolumn{2}{|c|}{2}} & \multirow[t]{3}{*}{3} & 4 & 5 \\
\hline & Capital humano & & & & 3 & 4 \\
\hline & Gestão da mudança & & & & & \\
\hline
\end{tabular}

Fonte: Valdés e colaboradores (2011). 


\section{Metodologia}

Com o objetivo de analisar os sites envolvidos neste estudo, foi realizada uma pesquisa qualitativa, na qual os dados foram coletados por meio de uma análise de conteúdo, que, segundo Krippendorff (2004), é uma técnica de pesquisa por meio da qual é possível fazer inferências válidas de textos para os contextos desejados.

Para realização da pesquisa, foi elaborado o protocolo de acordo com Luna Fo (1998), detalhado no quadro 4.

\section{Quadro 4 \\ Protocolo de pesquisa}

\begin{tabular}{|c|c|}
\hline $\begin{array}{l}\text { Revisão de } \\
\text { Literatura }\end{array}$ & $\begin{array}{l}\text { Focou principalmente a questão dos serviços eletrônicos e as formas de aferição dos usuários dos } \\
\text { serviços. }\end{array}$ \\
\hline $\begin{array}{l}\text { Questão do } \\
\text { Estudo }\end{array}$ & $\begin{array}{l}\text { A questão principal do estudo foi uma avaliação dos serviços eletrônicos disponibilizados pelos principais } \\
\text { sites estaduais no Brasil. }\end{array}$ \\
\hline $\begin{array}{l}\text { Seleção da } \\
\text { Amostra }\end{array}$ & $\begin{array}{l}\text { Foi realizada considerando-se todos os estados brasileiros dos quais foram selecionados os cinco (SP, } \\
\text { MG, RS, PR, SC) que representavam } 50 \% \text { dos bytes transmitidos. }\end{array}$ \\
\hline $\begin{array}{l}\text { Desenho do } \\
\text { Estudo }\end{array}$ & $\begin{array}{l}\text { Foi realizado um estudo observacional analítico, pois se tentou avaliar uma situação e seus processos } \\
\text { envolvidos. Neste sentido, foram coletadas as principais telas dos sites de e-gov que foram analisados. }\end{array}$ \\
\hline Análise dos Dados & $\begin{array}{l}\text { Utilizou-se a análise de conteúdo, por meio das dimensões (site, sinergia, eficiência, realização, } \\
\text { disponibilidade do sistema e privacidade). Feita esta classificação, foram atribuídas notas de } 1 \text { a } \\
5 \text { conforme fosse identificada a presença ou a ausência do elemento estudado e o seu grau de } \\
\text { desenvolvimento, partindo de } 1 \text { "Nada consta" ao } 5 \text { "Muito desenvolvido". }\end{array}$ \\
\hline
\end{tabular}

Fonte: Elaborado pelos autores a partir de Luna Fo (1998).

A técnica utilizada por esta pesquisa na análise de conteúdo foi a análise temática ou categorial por ser a que mais se adaptou à análise do conteúdo dos sites envolvidos neste estudo. Esta escolha é respaldada por Bardin (2011), que afirmou que esse tipo de análise é indicado para situações em que se utilizem peneiras de análise que remetam para uma variável, e que a escolha das unidades de registro e de contexto deve responder de maneira pertinente em relação às características da matéria e em face dos objetivos da análise.

Para a coleta dos dados por meio da análise de conteúdo, foram seguidas as etapas descritas por Bardin (2011), que são a pré-análise, a análise do material e o tratamento dos resultados, a inferência e a interpretação.

Como o objetivo desta etapa da pesquisa foi avaliar os serviços de sites de e-gov oferecidos pelos governos pesquisados, os documentos analisados foram os sites onde esses serviços são disponibilizados. 


\section{Resultados}

Para a organização da codificação dos dados, foram realizadas as seguintes escolhas indicadas por Bardin (2011):

- O recorte, que diz respeito às escolhas das unidades e que neste estudo podem ser classificadas como documentos, uma vez que os objetos analisados foram os sites;

- Classificação dos dados em categorias, denominadas "Dimensões", que se referem às dimensões que compõem o modelo proposto para análise dos serviços de sites de e-gov;

- A enumeração que envolve as escolhas das regras de contagem. Neste caso, foi a presença ou a ausência das dimensões a serem avaliadas neste estudo, atribuindo-se um nível de desenvolvimento (conjunto de notas de 1 a 5 a cada um dos elementos analisados).

Deste modo, foi possível construir o quadro 5, onde o modelo proposto neste estudo serviu de base para a análise. Os atributos do modelo proposto foram avaliados utilizando-se uma escala de cinco pontos, onde 1 representa "Nada consta"; 2 "Baixa ocorrência"; 3 "Funcionamento básico"; 4 "Desenvolvido" e 5 "Muito desenvolvido".

A partir do quadro 5 , foi possível verificar que, em relação ao primeiro atributo avaliado da dimensão Eficiência, os sites não apresentaram problemas de velocidade de acesso, o que mostra que estes sites conseguiram utilizar com eficiência os recursos multimídia sem comprometer a velocidade de acesso.

Quadro 5

Análise de conteúdo dos sites pesquisados

\begin{tabular}{|c|c|c|c|c|c|c|}
\hline DIMENSÕES & ATRIBUTOS & $\begin{array}{c}\text { Poupa- } \\
\text { Tempo } \\
\text { (SP) }\end{array}$ & $\begin{array}{l}\text { UAI } \\
(\mathrm{MG})\end{array}$ & $\begin{array}{l}\text { E-Paraná } \\
\text { (PR) }\end{array}$ & $\begin{array}{l}\text { Tudo- } \\
\text { Fácil } \\
\text { (RS) }\end{array}$ & $\begin{array}{c}\text { Site do } \\
\text { Estado } \\
\text { (SC) }\end{array}$ \\
\hline \multirow[t]{6}{*}{ Eficiência } & Tem velocidade de acesso & 5 & 5 & 5 & 5 & 5 \\
\hline & É fácil usar o site & 4 & 3 & 4 & 5 & 4 \\
\hline & Facilidade de interação & 5 & 5 & 2 & 2 & 2 \\
\hline & Conveniência de uso & 3 & 3 & 3 & 3 & 3 \\
\hline & Serviços personalizados & 1 & 1 & 1 & 1 & 1 \\
\hline & Serviços de qualidade & 2 & 1 & 1 & 2 & 3 \\
\hline \multicolumn{2}{|c|}{ Média dos atributos da dimensão Eficiência } & 3,33 & 3,00 & 2,67 & 3,00 & 3,00 \\
\hline \multirow[t]{3}{*}{ Realização } & $\begin{array}{l}\text { O site apresenta claramente os serviços } \\
\text { oferecidos }\end{array}$ & 4 & 5 & 5 & 5 & 4 \\
\hline & O site cumpre com os serviços oferecidos & 2 & 1 & 1 & 2 & 3 \\
\hline & $\begin{array}{l}\text { O site permite acompanhar processos em } \\
\text { andamento }\end{array}$ & 1 & 3 & 1 & 1 & 3 \\
\hline \multicolumn{2}{|c|}{ Média dos atributos da dimensão Realização } & 2,33 & 3,00 & 2,33 & 2,67 & 3,33 \\
\hline
\end{tabular}




\begin{tabular}{|c|c|c|c|c|c|c|}
\hline DIMENSÕES & ATRIBUTOS & $\begin{array}{l}\text { Poupa- } \\
\text { Tempo } \\
\text { (SP) }\end{array}$ & $\begin{array}{l}\text { UAI } \\
(\mathrm{MG})\end{array}$ & $\begin{array}{l}\text { E-Paraná } \\
\text { (PR) }\end{array}$ & $\begin{array}{l}\text { Tudo- } \\
\text { Fácil } \\
\text { (RS) }\end{array}$ & $\begin{array}{c}\text { Site do } \\
\text { Estado } \\
\text { (SC) }\end{array}$ \\
\hline \multirow[t]{3}{*}{$\begin{array}{l}\text { Disponibili- } \\
\text { dade }\end{array}$} & $\begin{array}{l}\text { O site não apresenta problemas de } \\
\text { indisponibilidade }\end{array}$ & 5 & 5 & 5 & 5 & 5 \\
\hline & Os links funcionam corretamente & 5 & 5 & 5 & 4 & 2 \\
\hline & O site não para (trava) durante o uso & 5 & 5 & 5 & 5 & 5 \\
\hline \multicolumn{2}{|c|}{ Média dos atributos da dimensão Disponibilidade } & 5,00 & 5,00 & 5,00 & 4,67 & 4,00 \\
\hline \multirow[t]{3}{*}{ Privacidade } & As transações exigem senhas & 1 & 1 & 1 & 1 & 1 \\
\hline & $\begin{array}{l}\text { O site disponibiliza política de segurança e } \\
\text { privacidade }\end{array}$ & 1 & 5 & 1 & 1 & 1 \\
\hline & $\begin{array}{l}\text { O site sinaliza a existência de mecanismos de } \\
\text { segurança }\end{array}$ & 1 & 1 & 1 & 1 & 1 \\
\hline \multicolumn{2}{|c|}{ Média dos atributos da dimensão Privacidade } & 1,00 & 2,33 & 1,00 & 1,00 & 1,00 \\
\hline \multirow[t]{3}{*}{ Site } & O site oferece o que era esperado & 2 & 3 & 2 & 2 & 2 \\
\hline & O site motiva o cidadão a voltar & 1 & 1 & 1 & 1 & 1 \\
\hline & O site estimula o cidadão a utilizá-lo & 1 & 1 & 1 & 1 & 1 \\
\hline \multicolumn{2}{|c|}{ Média dos atributos da dimensão Site } & 1,33 & 1,67 & 1,33 & 1,33 & 1,33 \\
\hline \multirow[t]{4}{*}{ Sinergia } & O site fornece informações de qualidade & 4 & 5 & 5 & 5 & 4 \\
\hline & O site fornece opções de comunicação & 4 & 4 & 5 & 3 & 4 \\
\hline & As opções de comunicação funcionam & 5 & 5 & 5 & 5 & 5 \\
\hline & O site disponibiliza FAQ & 5 & 5 & 1 & 1 & 1 \\
\hline \multicolumn{2}{|c|}{ Média dos atributos da dimensão Sinergia } & 4,50 & 4,75 & 4,00 & 3,50 & 3,50 \\
\hline
\end{tabular}

Fonte: Elaborado pelos autores.

Quanto à facilidade de usar os sites, algumas considerações precisam ser feitas:

1. o site do PoupaTempo poderia oferecer maior facilidade de uso se agrupasse os serviços e as informações de acordo com as características dos usuários (cidadãos, empresas, servidores públicos);

2. a facilidade de uso do site UAI foi prejudicada pelo fato de o mesmo direcionar suas opções ao site oficial do governo, onde o usuário novamente precisava procurar e solicitar o que desejava;

3. quanto ao E-Paraná, a facilidade de uso ficou comprometida pela utilização de um site unificado com o governo estadual, o que faz com que o mesmo tenha muita informação em uma mesma tela; e

4. no portal do estado de Santa Catarina, por vezes, o cidadão, após fornecer algumas informações, era direcionado a outro site onde o serviço já solicitado no portal anterior tinha de ser novamente procurado, solicitado e as informações mais uma vez fornecidas. 
Os recursos de interação, que visam a troca de informações e experiências entre indivíduos com interesses comuns, estavam presentes nos sites do programa paulista por meio de Twitter $^{8}$ e Blog 9 do próprio PoupaTempo e, no do governo mineiro, por meio de Twitter próprio, além de disponibilização de fotos e vídeos por meio de Fickr ${ }^{10}$ e YouTube. ${ }^{11}$ Os outros sites disponibilizavam links para as redes sociais, mas não possuíam páginas próprias nestas redes.

Os sites oferecem a seus usuários a conveniência de estarem disponíveis sete dias por semana, 24 horas por dia e em qualquer lugar que exista uma conexão com a internet. Essa conveniência também era oferecida pelos sites pesquisados, uma vez que os cidadãos podiam buscar o de que necessitavam sem se limitarem aos horários de funcionamento dos órgãos públicos, sem ter de se locomover até um desses lugares, sem ter de esperar na fila para serem atendidos, o que representa uma economia de tempo e de dinheiro. Porém essa conveniência ficou limitada à restrita disponibilização dos serviços aos cidadãos nos sites analisados.

Em relação à avaliação dos serviços, foi possível concluir que os sites analisados eram quase que totalmente informativos: apenas os sites do PoupaTempo e do TudoFácil ofereciam o serviço de agendar para ser atendido em uma de suas unidades físicas, um serviço limitado porque não substituía a execução de um serviço em seu modo tradicional.

Na página do PoupaTempo existia um link para a página da Secretaria da Segurança Pública, onde era possível solicitar e validar o atestado de antecedentes criminais. No site do estado de Santa Catarina, era possível imprimir guias para recolhimento de taxas e impostos e, também, fazer consultas como multas de trânsitos, resultados de provas de habilitação e horários e valores de ônibus intermunicipais. Nenhum dos sites pesquisados oferecia serviços personalizados. Como os sites não ofereciam serviços personalizados que pudessem substituir os serviços oferecidos pelo modo tradicional, os mesmos somente foram avaliados pelo único serviço oferecido (agendamento), prejudicando uma análise de outras possibilidades de serviços.

A média dos atributos que compõem a dimensão Eficiência atingiu os três pontos em quatro dos cinco sites analisados, ficando apenas o site do estado do Paraná um pouco abaixo deste número. Os atributos que contribuíram de modo positivo para a média desta dimensão foram velocidade de acesso e facilidade de utilizar o site. Entretanto, os atributos serviços personalizados e serviços de qualidade contribuíram para diminuir o valor da média alcançada pela dimensão, demonstrando serem importantes pontos de melhoria.

Nos sites referentes aos estados de Minas Gerais, Paraná e Rio Grande do Sul, os serviços oferecidos eram apresentados de acordo com as características de seus usuários (Cidadãos, Empresas, Servidores Públicos), o que facilitava o acesso aos mesmos. O site de Santa Catarina apresentava seus serviços classificados em Serviços On-line, Serviços aos Cidadãos,

\footnotetext{
${ }^{8}$ Rede de informação em tempo real composta por mensagens com até 140 caracteres (Twitter, 2011).

${ }^{9}$ PA frequent, chronological publication of personal thoughts and Web links. Publicações frequentes e cronológicas de pensamentos pessoais e links da Web (Blog, 2011).

${ }^{10}$ É um site que hospeda e partilha imagens fotográficas (e eventualmente de outros tipos de documentos gráficos, como desenhos e ilustrações), caracterizado também como rede social (Flickr, 2011).

${ }^{11}$ Site que permite que seus usuários carreguem e compartilhem vídeos em formato digital (YouTube, 2011).
} 
Outros Serviços e Consultas para Servidores, porém os mesmos serviços que estavam no grupo de Serviços On-line encontravam-se também na classe dos Serviços aos Cidadãos. Além disso, serviços como Licitações e Pregões encontravam-se em Serviços aos Cidadãos. O site do PoupaTempo apresentava seus serviços sem uma classificação formalmente disponibilizada aos seus usuários.

Para avaliar em que medida o site cumpre com o que promete aos seus usuários, seria necessário que os sites divulgassem quais são seus objetivos e suas principais atribuições, o que não aconteceu em nenhum dos sites analisados. A ausência destas informações pode gerar uma percepção ruim por parte dos usuários, que podem criar uma falsa perspectiva de conseguir realizar vários serviços por meio dessas páginas. Conforme descrito anteriormente, o governo do Estado de Minas Gerais desenvolveu seu portal para ser um balcão de informações e não para prestação de serviços e, como tal, o site mineiro cumpria com suas atribuições, porém essas informações deveriam ser fornecidas ao público que visita a página em questão. Se o objetivo dos sites analisados fosse informar seus cidadãos, pode-se afirmar que todos cumpririam com seus propósitos, porém, se o objetivo fosse disponibilizar em meio eletrônico os serviços oferecidos em unidades físicas, o mesmo estaria distante de ser atingido.

Em relação ao atributo de permitir acompanhar processos em andamento, o site do UAI direcionava todos os seus links para o site do governo mineiro e este oferecia a opção de acompanhar protocolos. Quando solicitado o serviço de boletim de ocorrência, o site de Santa Catarina direcionava para o site da polícia civil estadual que permitia consultar os registros de ocorrência. Nenhum outro atributo de acompanhamento foi encontrado nos demais sites analisados.

Ao analisar a média dos atributos da dimensão Realização (apresentar claramente os serviços oferecidos, cumprir com os serviços oferecidos e permitir acompanhar processos em andamento), foi possível verificar que, de modo geral, os sites cumpriram a função de apresentar, de modo claro, os serviços que disponibilizam. Contudo, existiram lacunas que deveriam ser trabalhadas em relação ao cumprimento dos serviços oferecidos e ao acompanhamento de processos em andamento.

Para que os usuários consigam ter acesso às informações e aos serviços que desejam, é essencial que os sites e seus links, atributos da dimensão Disponibilidade, funcionem corretamente. Nesta pesquisa, os sites analisados, bem como os links disponibilizados pelos mesmos, foram testados diversas vezes, em dias distintos, incluindo dias úteis, finais de semana e feriados e também em horários variados, e em nenhum destes testes apresentaram problemas que impedissem o acesso a eles ou a suas opções, com exceção da opção "Como chegar ao TudoFácil?" que não funcionava. Uma ressalva deve ser feita quanto ao site de Santa Catarina que apresentou 11 links que não possibilitaram o acesso.

Durante a realização das análises e testes, não houve nenhum registro de travamento de alguma transação, o que pode demonstrar preocupação dessas organizações em manter seus portais com um equilíbrio adequado de recursos tecnológicos que não inviabilizem a utilização dos mesmos. É importante destacar que para tais análises foram utilizados os mesmos recursos tecnológicos (computador, provedor de internet), pois, conforme afirmaram Para- 
suraman, Zeithaml e Malhotra (2005), as empresas não podem ter controle total sobre esse atributo porque o equipamento nas instalações do cliente (por exemplo, o tipo de computador e ligação à internet) também é susceptível de afetar o desempenho.

O funcionamento correto dos sites, de seus links e de suas transações foi demonstrado pelas altas médias alcançadas dos atributos da dimensão Disponibilidade. Esses valores podem sinalizar elementos do governo eletrônico que estão sob o domínio de seus administradores.

Os sites analisados não disponibilizavam informações confidenciais ou pessoais e também não as solicitavam aos seus usuários. Deste modo, não havia nenhuma transação que exigisse senha ou site que apresentasse a existência de mecanismos de segurança. O site de Minas Gerais era o único que oferecia esclarecimentos quanto às responsabilidades e divulgações de informações em "Aspectos Legais e Responsabilidades".

As baixas médias dos atributos da dimensão Privacidade (exigência de senhas para realizar transações, política de segurança e privacidade disponível e existência de mecanismos de segurança) não devem ser analisadas como uma falta de preocupação com questões importantes como essas. Deve-se considerar o fato de que os sites analisados não disponibilizavam transações que solicitavam ou ofereciam informações confidenciais. Neste sentido, a existência de tais mecanismos de segurança e privacidade pode ser questionável.

Conforme afirmaram Kumar e colaboradores (2007), o objetivo principal dos sites de e-gov deve ser seu uso frequente por parte dos cidadãos para obter informações, interagir e realizar transações com o governo. Para aumentar a frequência de uso, os sites deveriam manter seus usuários satisfeitos e motivados por meio dos atributos da dimensão Site. Nos sites analisados, não foi verificada a utilização de nenhum recurso que pudesse motivar o uso ou o retorno do cidadão.

Um recurso utilizado em sites de comércio eletrônico são as pesquisas de satisfação para verificar, dentre outras coisas, se o site está oferecendo o que seu consumidor realmente espera. Esse recurso foi encontrado apenas no site do governo de Minas Gerais e, portanto, o mesmo servia para avaliar todos os aspectos deste governo e não somente do programa UAI.

A falta de recursos motivacionais nos sites analisados foi refletida na baixa média dos atributos da dimensão Site (oferecer o que o cidadão espera, motivar o cidadão a voltar a utilizar o site mais vezes, estimular o cidadão a fazer uso do site). Essa situação deve ser motivo de preocupação porque nenhum dos programas avaliados conseguiu atingir sequer a média de dois pontos e, conforme relatado no referencial teórico, a falta desses recursos pode inviabilizar a manutenção desses sites devido à falta de acessos.

Como os sites analisados representavam programas que ofereciam centenas de serviços públicos e, por meio destas páginas, os cidadãos não conseguiam realizar quase que nenhum serviço, é possível concluir que estes sites ainda não conseguiam oferecer o que seus cidadãos esperavam, com exceção ao UAI, que se propôs a ser um balcão de informações.

Em contraste com a escassez de serviços, os sites ofereciam um grande volume de informações e estas eram bem estruturadas: com exceção da página do PoupaTempo, as informações eram agrupadas de acordo com as necessidades de seus usuários, como Cidadãos, Empresas e Servidores Públicos. Além disso, nestes grupos, as informações eram ainda dis- 
ponibilizadas de acordo com assuntos de interesse como Água, Educação e Transporte. Essa estrutura facilita sobremaneira a busca e o acesso às mesmas.

Além da preocupação com a estrutura das informações, foi possível verificar que os sites, exceto o do PoupaTempo e o de Santa Catarina, ofereciam recursos de busca.

Outra exceção negativa do PoupaTempo em relação à qualidade das informações: foi o único site onde foram encontradas informações desatualizadas. A opção "Na Imprensa" não trazia nenhuma notícia do ano de 2011. Em contraste, o portal de Santa Catarina disponibilizava informações em excesso: 50 páginas de "Últimas Notícias".

Com exceção do site do TudoFácil, os sites disponibilizavam mais de uma opção de comunicação com seus usuários.

A comunicação por e-mail foi a opção escolhida por todos os sites, seguida pelo telefone. Deve-se ressaltar que o maior número de opções de comunicação com o usuário era oferecido pelo site unificado do estado do Paraná, talvez justamente por esta unificação. Em alguns sites, como no PoupaTempo e no UAI, foi possível a comunicação por e-mail, onde o sistema de resposta automática funcionou de modo correto. No site do estado do Paraná foi possível utilizar a comunicação por meio do chat on-line, que também transcorreu sem nenhuma intercorrência.

A opção de Ouvidoria era disponibilizada pelo PoupaTempo, pelo site de Santa Catarina e do governo do Paraná, onde o E-Paraná estava unificado. O site do PoupaTempo e o site do governo de Minas Gerais foram os únicos que possuíam FAQ, que é uma ferramenta de grande auxílio ao usuário.

Ao analisar as médias dos atributos da dimensão Sinergia foi possível verificar que estas não foram superiores a quatro somente nos sites que não disponibilizavam FAQ (Paraná, Rio Grande do Sul e Santa Catarina), destacando, desta maneira, um elemento que deve ser incorporado nestes programas para que os mesmos possam ser mais bem avaliados.

Após a realização destas análises, é possível, a partir das definições do modelo de nível de maturidade de e-gov de Valdés e colaboradores (2011), concluir que os sites analisados neste estudo encontravam-se entre os níveis dois e três de maturidade.

\section{Considerações finais}

Com o objetivo de propor um modelo para analisar os serviços dos sites de e-gov, este estudo se propôs a realizar uma análise de conteúdo de um site de governo eletrônico de cada um dos cinco estados brasileiros que juntos representam 50\% dos sites de e-gov nacional, que são os estados de São Paulo, Minas Gerais, Paraná, Rio Grande do Sul e Santa Catarina.

O principal ponto a ser destacado é que o modelo proposto se mostrou um instrumento adequado para analisar os serviços de sites de e-gov, de fácil aplicação.

Com a aplicação do modelo, importantes pontos foram destacados:

จ A necessidade de disponibilizar mais serviços; 
、 A importância da existência dos aspectos relacionados à segurança e à privacidade;

v Fazer uso de recursos que possam motivar e estimular os usuários a utilizarem o site com frequência.

As principais contribuições deste estudo para a área acadêmica estão relacionadas com o desenvolvimento de um modelo que permite analisar os serviços de sites de governo eletrônico e que agrega, de modo inovador e complementar, aspectos considerados por importantes estudos realizados nesta área, tendo em conta o ponto de vista do cidadão, usuário dos serviços avaliados.

Em relação à área gerencial, é possível afirmar que este estudo contribuiu com o desenvolvimento de um modelo de fácil aplicação, para analisar os serviços de sites de e-gov sob a ótica dos usuários destes serviços, auxiliando-os a identificar aspectos importantes para os usuários dos serviços de e-gov que poderiam não estar recebendo a devida atenção de seus gestores.

Uma limitação deste estudo se refere aos estados selecionados bem como aos programas de e-gov pesquisados em cada estado, ou seja, foram estudados cinco estados (São Paulo, Minas Gerais, Paraná, Rio Grande do Sul e Santa Catarina) e um site de serviços de e-gov de cada um desses estados (PoupaTempo, UAI, E-Paraná, TudoFácil e o site do governo do estado de Santa Catarina). Como foi relatado anteriormente neste estudo, existem outras importantes iniciativas de serviços de e-gov nestes estados que poderiam fornecer contribuições valiosas.

Embora os estados analisados neste estudo representem 50\% dos sites de e-gov nacionais, existem muitas iniciativas em outros estados brasileiros e outras iniciativas nas esferas federais e municipais que poderiam ser analisadas.

Finalmente, outra limitação diz respeito ao próprio objeto de estudo: sendo o governo eletrônico assunto recente, dinâmico e multidisciplinar, pode envolver áreas tão distintas e abrangentes que seria impossível, em um único estudo, cobrir de maneira eficiente todos os seus aspectos. Portanto, o estudo em questão teve seu foco nos serviços prestados aos cidadãos, ciente de que outros elementos devem ser estudados em pesquisas futuras.

Conforme relatado por várias pesquisas descritas neste estudo, o e-gov pode ser considerado um assunto recente e, portanto, representa uma área que carece de um maior número de estudos. Além disso, a maioria dos estudos disponíveis sobre e-gov, muitos dos quais foram aqui citados, tem seu foco na área técnica que envolve principalmente aspectos relacionados aos sistemas de informação. Desse modo, existe a necessidade de se desenvolverem pesquisas que foquem outros elementos contidos no e-gov como serviços, qualidade, satisfação, imagem, cidadãos, empresas e governo.

A pesquisa aqui desenvolvida também poderia ser aplicada em outros programas de e-gov dos estados aqui pesquisados bem como em programas de outros estados, municípios ou federações. 


\section{Referências}

ALMEIDA, Marcos O. Governo eletrônico no Brasil. In: CONGRESO INTERNACIONAL DEL CLAD SOBRE LA REFORMA DEL ESTADO Y DE LA ADMINISTRACIÓN PÚBLICA, VII, 2002, Lisboa.

ARAKAKI, Cristiane. O governo eletrônico como instrumento de aproximação do governo e o cidadão. Dissertação (mestrado) — Faculdade de Comunicação, Universidade de Brasília, Brasília, 2008.

BARDIN, Laurence. Análise de conteúdo. Tradução Luís Antero Reto e Augusto Pinheiro. São Paulo: Edições 70, 2011.

BLOG. Disponível em: <www.marketingterms.com/dictionary/blog/>. Acesso em: 23 nov. 2011.

COLLIER, Joel E.; BIENSTOCK, Carol C. Measuring service quality in e-retailing. Journal of Service Research, v. 8, n. 3, p. 260-275, Feb. 2006.

COMITÊ GESTOR DA INTERNET NO BRASIL (CGI.br). Dimensões e características da Web brasileira: um estudo do .gov.br. Disponível em: <www. W3c.br>. Acesso em: 19 maio 2011.

CONSTANTINIDES, Efthymios. From physical marketing to web marketing: the web-marketing mix. Proceedings of the 35th Hawaii International Conference on System Sciences, 2002a.

CONSTANTINIDES, Efthymios. Influencing the online consumer's behavior: the web experience. Internet Research, v. 14, n. 2, p. 111-126, 2004.

CONSTANTINIDES, Efthymios. The 4S web-marketing mix model. Electronic Commerce Research and Applications, v. 1, p. 57-76, 2002b.

COSTA, Frederico L. Brasil: 200 anos de Estado; 200 anos de administração pública; 200 anos de reformas. RAP — Revista de Administração Pública, v. 42, n. 5, p. 829-874, set./out. 2008.

DINIZ, Eduardo H. et al. O governo eletrônico no Brasil: perspectiva histórica a partir de um modelo estruturado de análise. RAP — Revista de Administração Pública, v. 43, n. 1, p. 23-48, jan./fev. 2009.

DINIZ, Eduardo H. Uso do comércio eletrônico em órgãos do governo. EAESP/FGV/NPP — Núcleo de Pesquisas e Publicações 1/100, Relatório de Pesquisa n. 18, 2000.

ENDLER, Antônio M. Governo eletrônico: a internet como ferramenta de gestão dos serviços públicos, REAd, Ed. 14, v. 6, n. 2, mar./abr. 2000. Disponível em: < http://read.adm.ufrgs.br/read14/artigo/artigo1.htm>. Acesso em: 22 maio 2011.

FERNANDES, Andréa G.; AFONSO, José Roberto R. e-governo no Brasil: experiências e perspectivas, Revista do BNDES, v. 8, n. 15, p. 21-64, jun. 2001.

FLICKR. Disponível em: <http://dicionario.babylon.com/flickr/>. Acesso em: 23 nov. 2011. 
GONÇALES, Marco A. D.; FERREIRA, Simone B. L. O comércio eletrônico a partir de ambientes públicos. Universidade Federal do Estado do Rio de Janeiro. Centro de Ciências Exatas e Tecnologia. Relatórios Técnicos do Departamento de Informática Aplicada, n. 4, 2010.

HOESCHL, Hugo C. Aplicações inteligentes para governo eletrônico. Pós-doutorado - Instituto de Governo Eletrônico, Inteligência Jurídica e Sistemas, Universidade Federal de Santa Catarina, 2003.

KRIPPENDORFF, Klaus. Content analysis: an introduction to its methodology. Thousand Oaks, Ca: Sage Publications, 2004.

KUMAR, Vinod et al. Factors for successful e-government adoption: a conceptual framework. Electronic Journal of e-Government, v. 5, n. 1, p. 63-76, 2007.

LIN, Hsiu-Fen. Empirically testing innovation characteristics and organizational learning capabilities in e-business implementation success. Internet Research, v. 18, n. 1, p. 60-78, 2008.

LUNA F ${ }^{\circ}$, Bráulio. Sequência básica na elaboração de protocolos de pesquisa. Arq. Bras. Cardiol., v. 71, n. 6, 735-740, 1998. Disponível em: <www.scielo.br/pdf/abc/v71n6/a01v71n6.pdf>. Acesso em: 8 mar. 2013.

McNEAL, Ramona S. et al. Innovating in digital government in the American states. Social Science Quarterly, v. 84, n. 1, p. 52-70, 2003.

OSBORNE, David; GAEBLER, Ted. Reinventando o governo: como o espírito empreendedor está transformando o setor público. Brasília: Enap, 1997.

PARASURAMAN, A.; ZEITHAML, Valarie A.; MALHOTRA, Arvind. E-S-QUAL a multiple-item scale for assessing electronic service quality. Journal of Service Research, v. 7, n. 3, p. 213-233, Feb. 2005.

PROFESSOR DIGITAL. Governo eletrônico. Disponível em: <www.professor.rs.gov.br/governoeletronico/>. Acesso em: 13 jul. 2011.

SANCHEZ, Oscar A.; ARAÚJO; Marcelo. O governo eletrônico no estado de São Paulo, Série Didática, n. 6, jul. 2003. Disponível em: <www.cedec.org.br/files_pdf/DIDATI7-GOV\%20ELETR.pdf>. Acesso em: 26 maio 2011.

TWITTER. Disponível em: <http://twitter.com/about>. Acesso em: 23 nov. 2011.

UAI. Unidade de Atendimento Integrado. Disponível em: <www.egov.mg.gov.br/UAI-2>. Acesso em: 15 jun. 2011.

VALDÉS, Gonzalo et al. Conception, development and implementation of an e-government maturity model in public agencies. Government Information Quarterly, v. 28, p. 176-187, 2011.

VILLELA, Renata M. Conteúdo, usabilidade e funcionalidade: três dimensões para a avaliação de portais estaduais de governo eletrônico na web. Dissertação (mestrado em ciência da informação) — Escola de Ciência da Informação, Universidade Federal de Minas Gerais, Belo Horizonte, 2003.

WANG, KanLiang; WANG, Yuan; YAO, JingTao. A comparative study on marketing mix models for digital products. In: Proceedings of the First International Workshop on Internet and network Economics (Wine'05). Hong Kong, Dec. 15-17, 2005. p. 660-669. 
WELCH, Eric W.; HINNANT, Charles C.; MOON, M. Jae. Linking citizen satisfaction with e-government and trust in government. Journal of Public Administration Research and Theory, v. 15, n. 3, p. 371-391, 2005.

WEST, Darrell M. E-government and the transformation of service delivery and citizen attitudes. Public Administration Review, v. 64, n. 1, p. 15-27, 2004.

YOUTUBE. Disponível em: <http://pt.wikipedia.org/wiki/YouTube> . Acesso em: 23 nov. 2011.

Ieda Pelógia Martins Damian é doutora em administração pela Faculdade de Economia, Administração e Contabilidade de Ribeirão Preto (FEA-RP/USP). E-mail: ieda.martins@bol.com.br.

Edgard Monforte Merlo é professor doutor da Faculdade de Economia, Administração e Contabilidade de Ribeirão Preto (FEA-RP/USP). E-mail: edgardmm@usp.br. 
